\section{Inauguraciones en el MNBA Santiago, Chile}

Una serie de celebraciones en el Museo Nacional de Bellas Artes es abordada desde el punto de vista del comer y el beber. La celebración posibilita el llevar a objetos de diseño la medida de dichos actos. Se mide el cuerpo y su estar de pie para generar alturas, posiciones, sustento y desplazamientos de los elementos.
A series of celebrations at the Fine Arts Museum in Santiago are approached from the perspective of eating and drinking The celebration makes possible the use of objects created specifically to accommodate certain actions. The standing body is measured to generate heights, positions, supports and displacements of the elements.

Ricardo Lang Profesor, Pontificia Universidad Católica de Valparaíso Silvia Arriagada Profesora, Pontificia Universidad Católica de Valparaíso
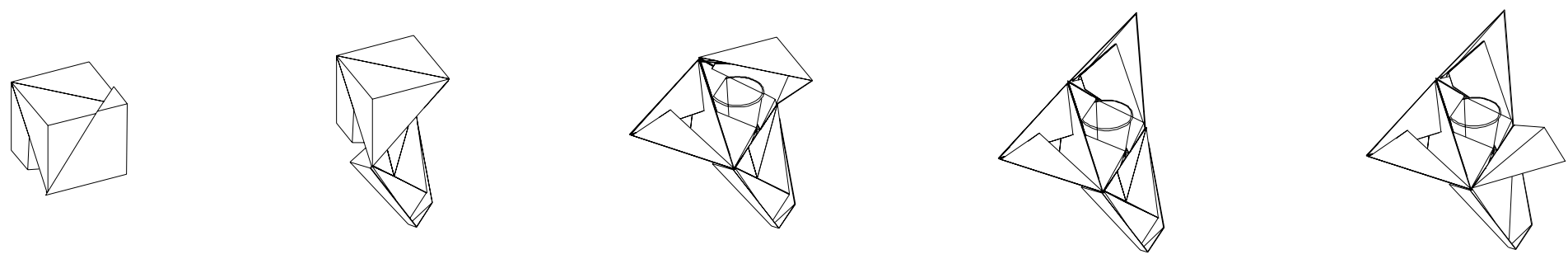

Quién de nosotros no acompañó a su madre o abuela en esas tardes de divertimento junto a la cocina, donde desde niño nos iban dando pequeñas tareas que hacíamos con gran devoción: partir un huevo -el escurridizo intento de separar la yema de la clara-, intentar batirlos, enmantequillar un molde, troquelar galletas o sopaipillas, despegar, levantar y volver a comprimir las caladas tramas, amasarlas nuevamente - cada vez menores-, hasta que la autorización llegaba para que, con un insignificante resto, pudiésemos realizar libremente el juego de nuevas proposiciones, nunca certeras.

Luego, el aparecer lento de los aromas que se extendían hasta espacios ajenos, verificando la presencia de aquella secreta y doméstica alquimia de sabores, ceñida a temperaturas y tiempos predestinados por esa herencia infatigable de secretos de familia, o bien, por rigurosos pasos impresos. Ese tiempo otorgado a la espera predisponía el ánimo al goce compartido de lo realizado: poner la mesa, vestirla, cubrirla de objetos y utensilios. En cada una de ellos, en los gestos y cuidados, hay un gran oficio, desde la tratada superficie del mantel, la delicada cerámica de la vajilla, los pulidos metales de la cuchillería, los destellantes cristales; en fin, nada al azar. Todo lo acontecido -incluido lo encomendado inicialmente como un divertimentodemostrará que la elocuencia de su final transita entre sabores elaborados, intangibles, sellados por manos de abuelas, de madres o bien de lugares, sabor primero que cala y se absorbe como primer canon irrenunciable.
A pocas décadas de ese infantil momento de ocio, se hace necesario remirarlo, volverse sobre él como experiencia y ocupación distraída y afanada. El diseño que llevamos adelante quiere dar sentido a la dispersión en la que tal momento aparece en nuestras vidas y añade a ese operar sensible y natural la posibilidad de hacer vigente la construcción del tiempo, no cualquiera, sino aquel que pulsa al objeto, al espacio y a los seres humanos para revelar la fiesta.

El encuentro festivo, jamás desprendido del comer y del beber - desde lo frugal a lo abundante-, ha de vérselas hoy con el diseño de los objetos, sabiendo de antemano que serán docenas, cientos o miles, con la figura de ellos en el espacio, con el acto que da forma a esa figura donde los seres humanos sin vacilación, celebran manifiestamente ese presente.

Es que es el acto del diseño que tiene la capacidad intrínseca de aunar la extrema necesidad con la máxima gratuidad. Concentrar en un acto tal dispersión y abrirse al espacio del regalo que da destino a ese momento ocioso, que nace de una poética que se detiene en la levedad presente en cada ocasión de obra efímera e inicial.

Este cada vez de los actos de celebración aquí expuestos siempre arroja una sentencia que nace indistintamente de una lectura de quienes son los otros, del espacio, de la materia puesta en juego, del comer, de la hora del día, del traslado y sus distancias, de los tiempos, de las manos ejecutantes y de los costos; o bien de ese diálogo entre idas y venidas que dilata la aparición del objeto para desatar que la ocasión sea única e irrepetible.

¿Cuál es la unidad que deja a cada ocasión con la medida de común unión?

Son verbos los que se anticipan a toda la acción que se desarrollará y que se debatirá en múltiples particularidades. Ellos dan figura al ofrecimiento, vagan por la forma inaparente que precisa de definiciones. Ese estado de contemplación no sólo alude al hacerse sino también a mirar lo hecho.

Así como ese primer momento en que el niño, la madre, la masa, el fuego, la mesa, los utensilios evocan lo gratuito de la condición humana; o como un barco navegando por el horizonte da cuenta de los mares y por ende del tamaño del mundo, así también estos actos del diseño, en su trato del tiempo y el espacio, hacen aparecer de tanto en tanto la gracia escondida en el género humano. ARQ

\section{Bibliografía sugerida}

Baixas, Isabel; Baixas, Juan Ignacio y Ricardo Lang. "Primera Bienal de Diseño 1991, Centro de Extensión Puc". $A R Q, N^{\circ} 19$, Materia. Ediciones ARQ Santiago, junio de 1992. / Lang, Ricardo. “Diseño de tres celebraciones". ARQ $\mathrm{N}^{\circ} 49$, Diseño en Chile. Ediciones ARQ, Santiago, diciembre de 2001. / Arriagada, Sylvia y Ricardo Lang. "Umbral de las dos orillas, Hermenegildo, Brasil”. ARQ, $\mathrm{N}^{\circ} 64$, Chile dentro $y$ fuera. Ediciones ARQ, Santiago, diciembre de 2006. 


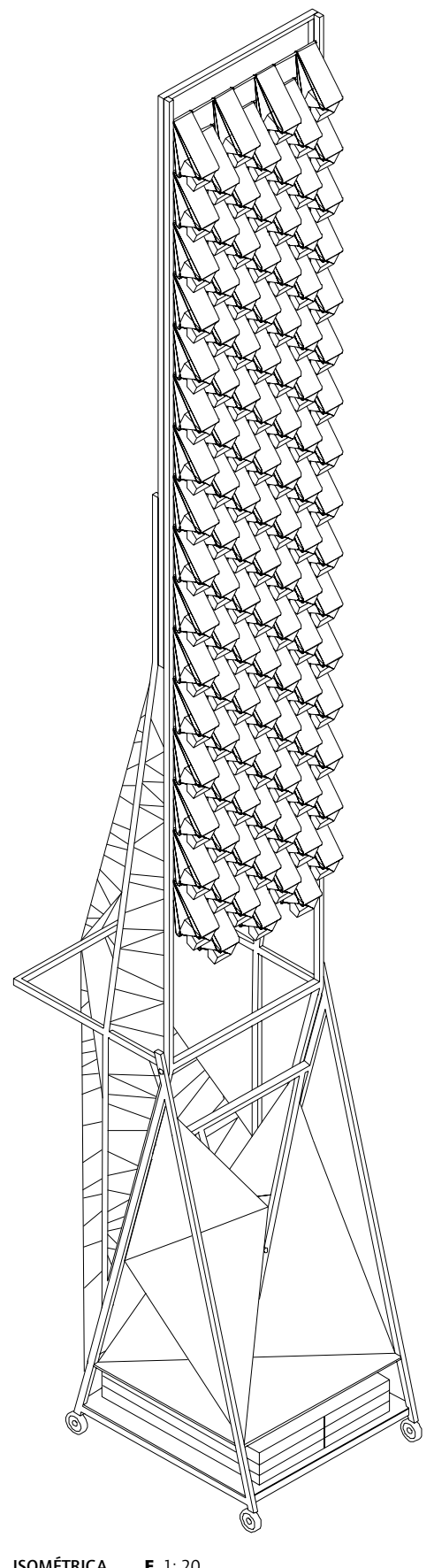

ISOMÉTRICA E 1:20

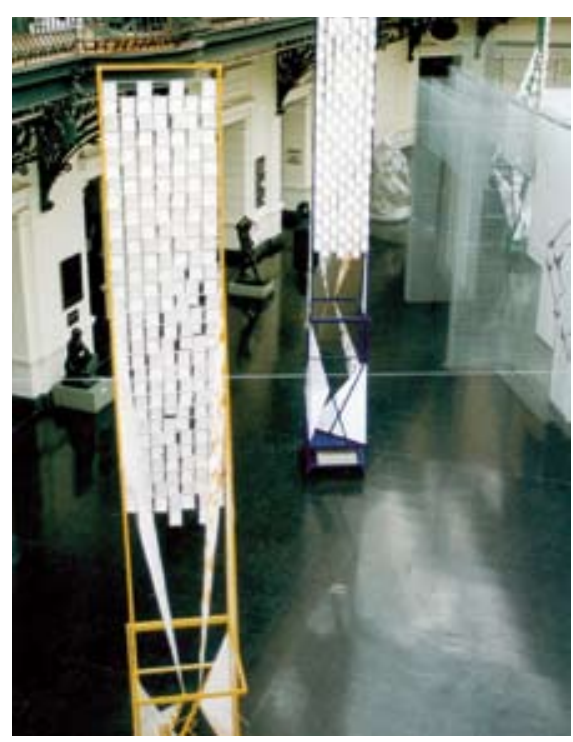

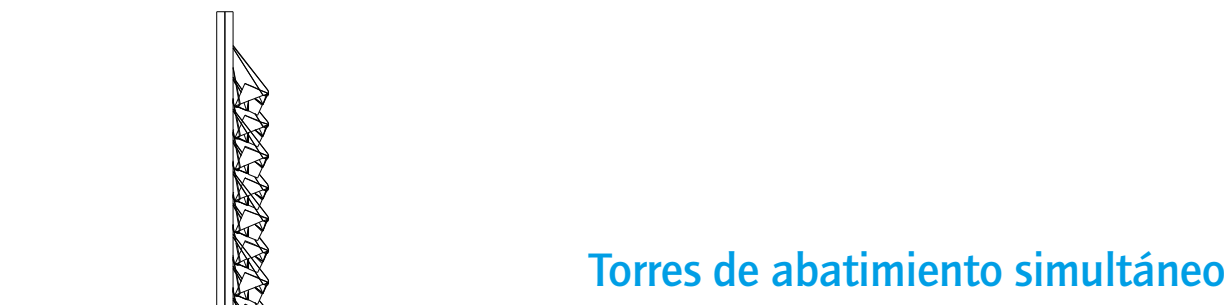

Unas manos ansiosas que desbarataron la conformación de nuestro cálculo en la inauguración de la Segunda Bienal de Diseño, en el Museo de Bellas Artes, fueron las que desataron esta nueva figura en la Bienal siguiente. Ahora, levantar los bocados en el espacio para evitar ese rapto, alejado e inalcanzable; por eso arriba, inequívocamente visibles y simultáneas al momento de ofrecer. Se parte de un espacio temporal que se inicia con la figura estática de unas torres erguidas, continuando con el abatimiento sincronizado de ellas deteniéndose a la altura de los brazos extendidos de los invitados, momento para que ellos desprendan las figuras cúbicas minuciosamente dispuestas por un tejido de papel. La figura cúbica proporciona a primera vista una cierta neutralidad que no advierte su contenido ni su uso. Al ser abierto produce un cambio radical en su geometría, haciendo aparecer inmediatamente el calce de un vaso a la mano. Elemento que da sentido y orden al posterior ejecutar de las manos.

\section{TORRES DE ABATIMIENTO SIMULTÁNEO}

INAUGURACIÓN TERCERA BIENAL DE DISEÑO

Organizador Pontificia Universidad Católica de Chile Ubicación Hall Central MNBA, Santiago, Chile Profesor Ricardo Lang

Colaboradores José Balcells, Alejandro Garretón

Materialidad soportes de contrapeso de hormigón, perfiles metálicos, tensores, alambre, placa MDF, papel estampado; porta cubos en tubo metálico, papel hilado y alambre; elemento portable en cartulina, vasos de polietileno transparente, papel adhesivo de color

Invitados 672 personas Fecha octubre de 1995 

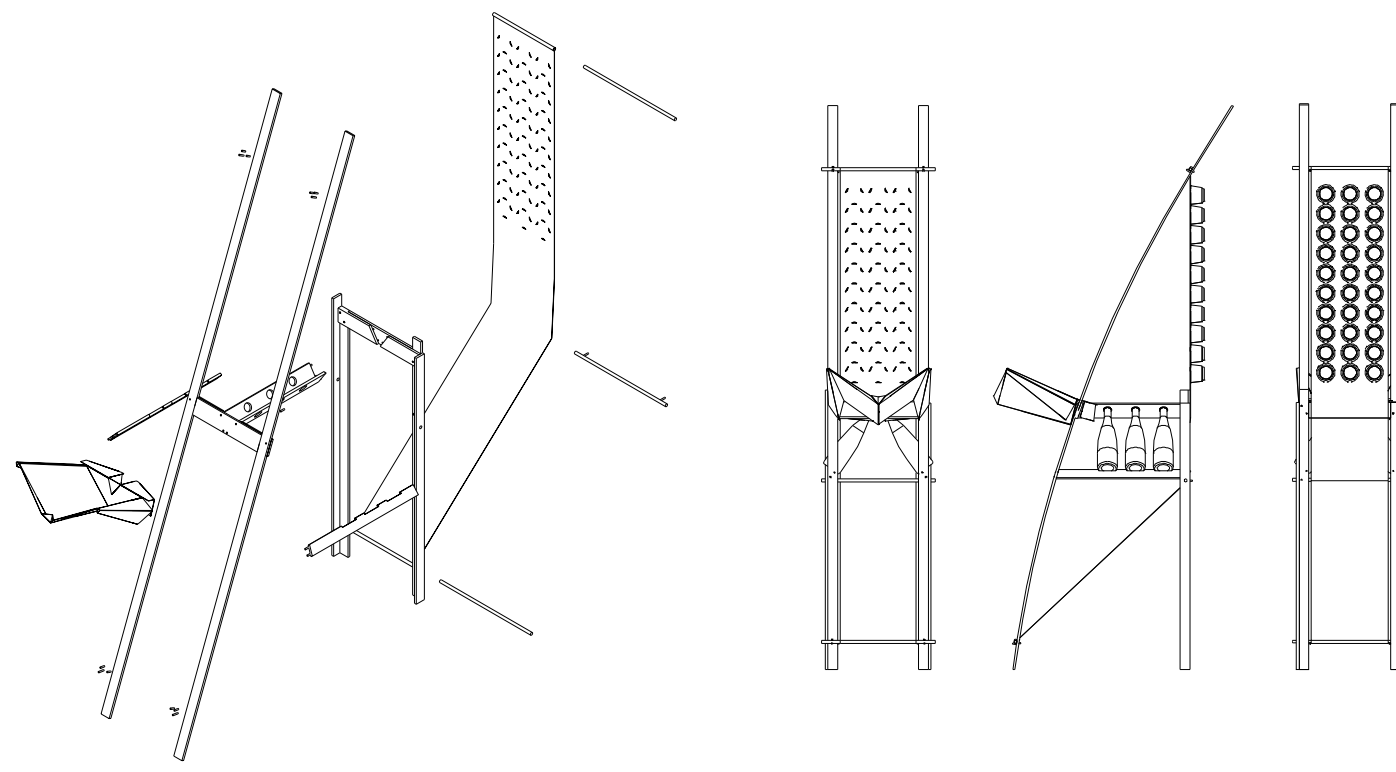

ELEVACIONES MOBILIARIO

E 1:20

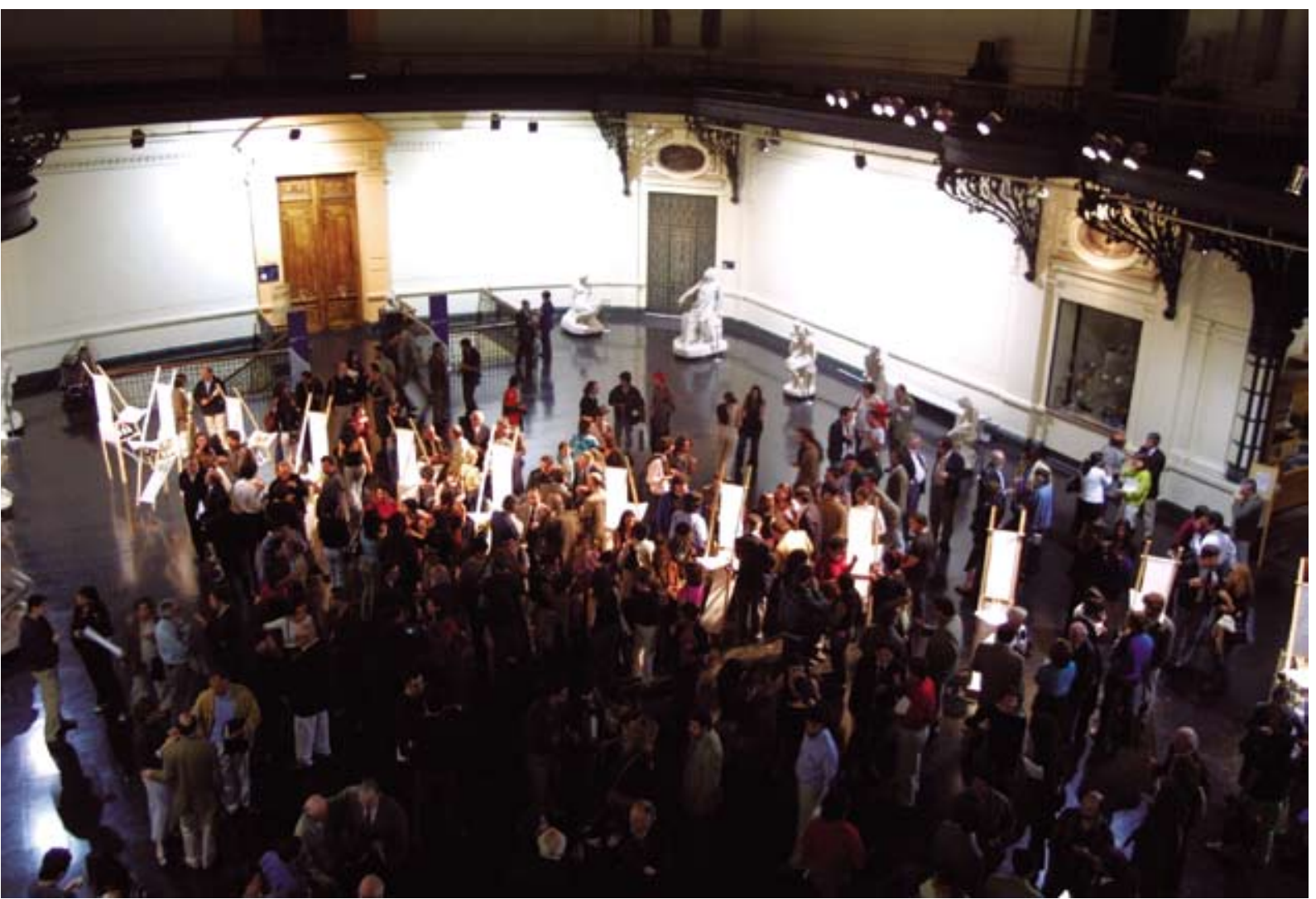

MOBILIARIO TRASLÚCIDO

LANZAMIENTO LIBRO AMEREIDA-PALLADIO

Organizador Escuela de Arquitectura y Diseño Pontificia Universidad Católica de Valparaíso Ubicación Hall Central MNBA, Santiago, Chile Profesor Ricardo Lang

Colaboradora Carolina Garrido

Materialidad soporte de portavasos de papel dia-

mante, estructura de terciado; portabocados en papel doble faz; pasadores de tarugos de madera; vasos de policarbonato blanco

Invitados 350 personas

Fecha septiembre de 2004

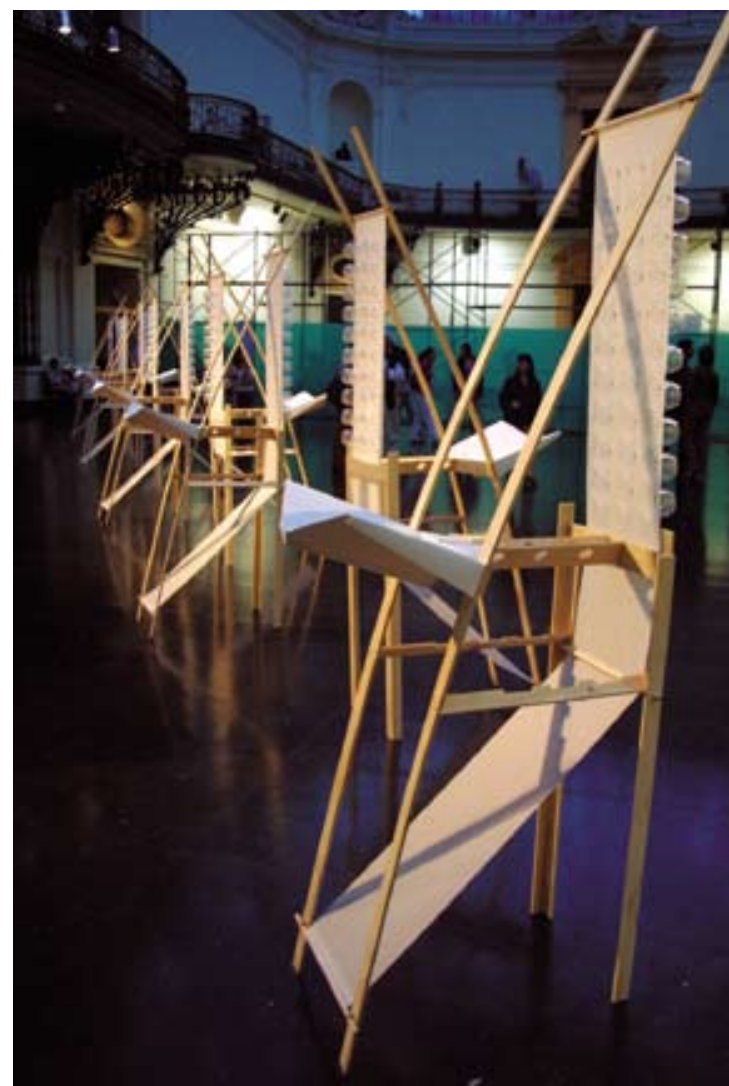




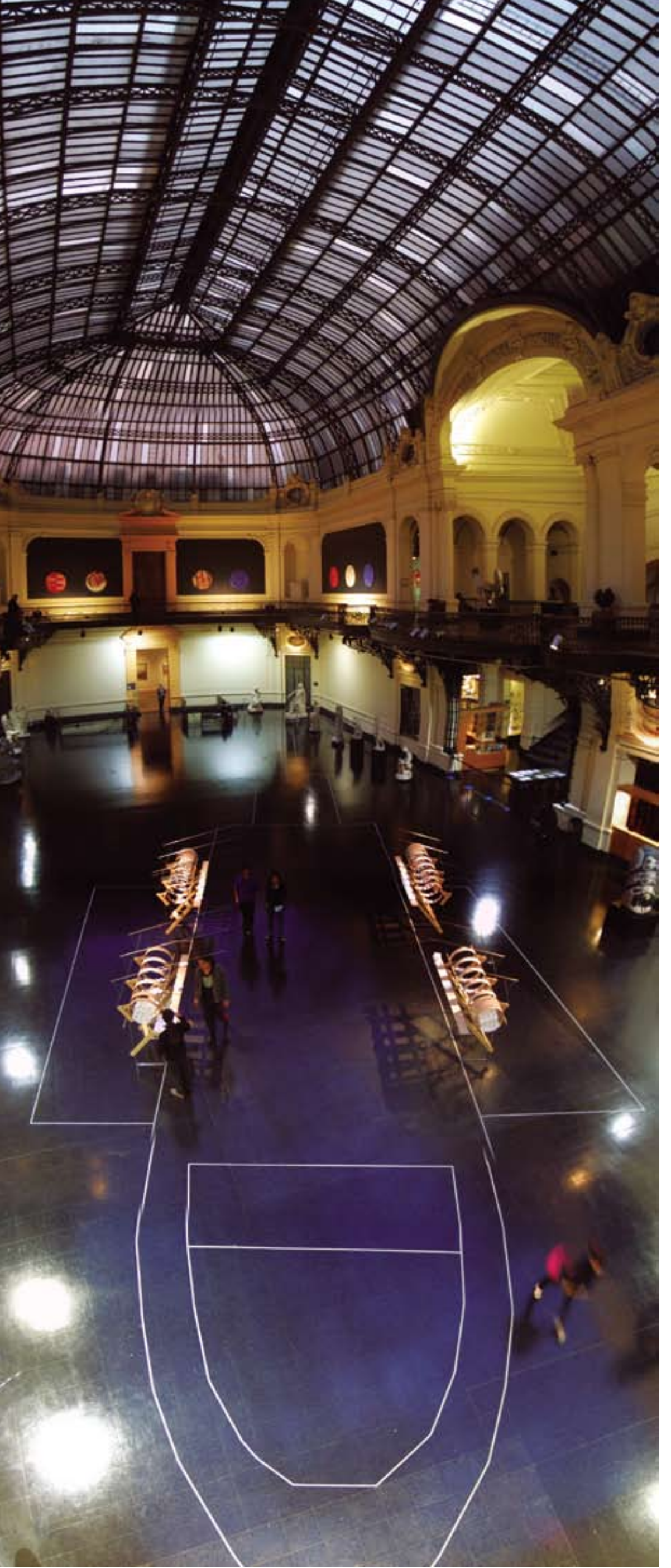

Mobiliario en dispersión

Fue el trazado de la embarcación Amereida, a escala 1:1 y que dibujamos en el negro suelo del Museo Nacional de Bellas Artes, el que nos permitió ver por primera vez la medida total de su despliegue. Sobre este trazado se hizo necesario ubicar elementos volumétricos que dieran cuenta de la habitabilidad presente y necesaria en toda embarcación mayor.

Cuatro cuerpos cilíndricos enfrentados de dos en dos se introdujeron a este trazado a la espera de la fiesta, simulando las balsas de salvamento apostadas permanentemente en las borda. De estos objetos desconocemos su despliegue, pues habitualmente permanecen a la espera de la ocasión que no siempre es agraciada pero sí agradecida.

Ellos en ese momento guardan, protegen y ocultan los contenidos del brindis, que se desplegaron por anillos en ofrecimientos reiterados.

\section{MOBILIARIO EN DISPERSIÓN}

LANZAMIENTO LIBRO LA EMBARCACIÓN AMEREIDA Y LA ÉPICA DE FUNDAR EL MAR PATAGÓNICO

Organizador Escuela de Arquitectura y Diseño Pontificia Universidad Católica de Valparaíso Ubicación Hall Central MNBA, Santiago, Chile Profesor Ricardo Lang

Colaboradores Silvia Arriagada, Felipe Cardemil, Manuel Sanfuentes Materialidad mesas de marcos metálicos, piezas MDF, prensa servilleta en alambre acerado, cubierta en cartulina doble faz, tubo de fierro, tensores de alambre; elemento portable compuesto por anillo en lámina MDF, tubos de aluminio, fijadores en cartulina, fijador de vasos en papel hilado, bandeja de cartulina doble faz Invitados 320 personas Fecha mayo de 2006

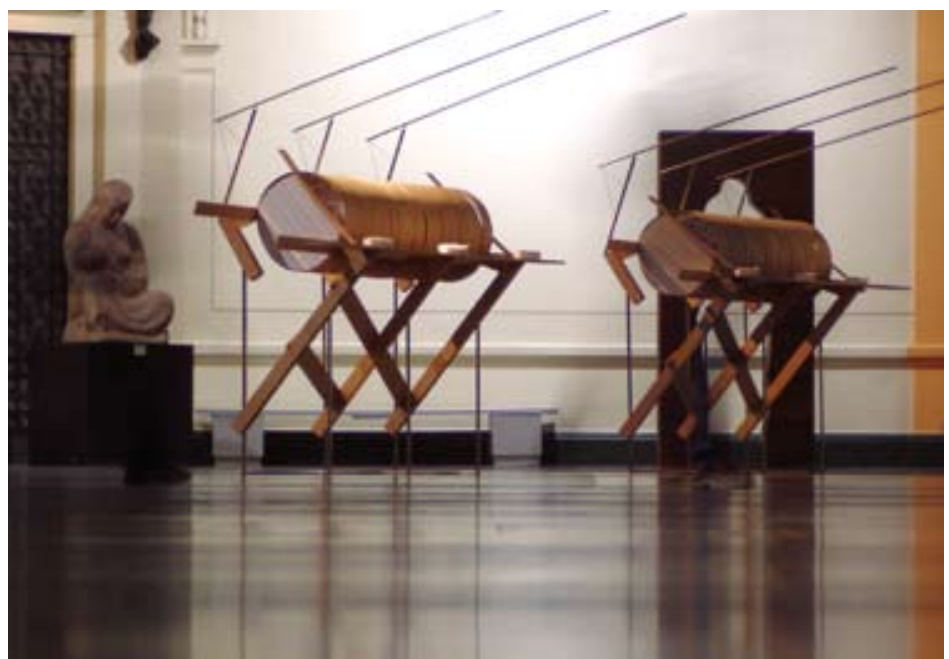




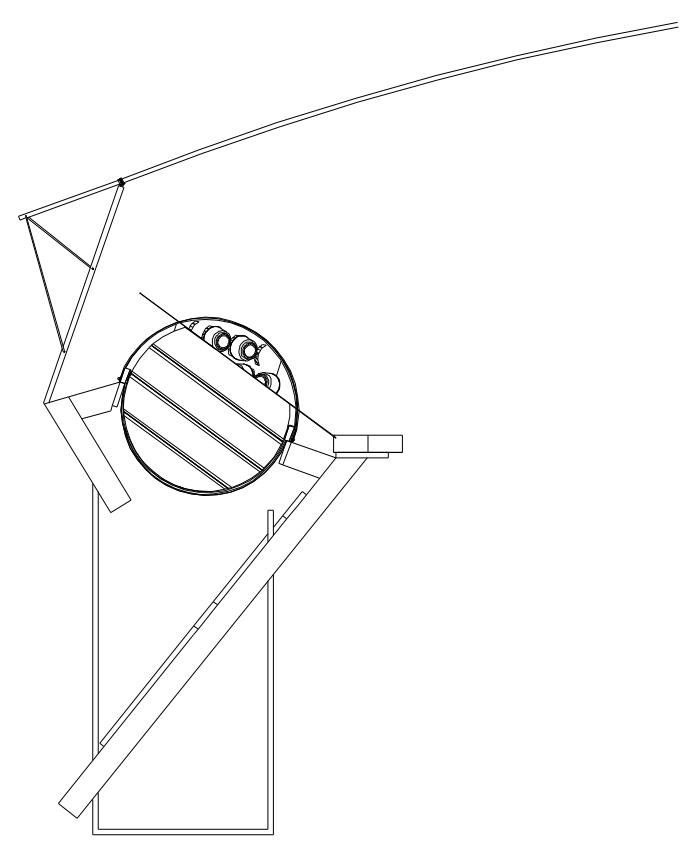

ELEVACIÓN SOPORTE Y CUERPO CILINDRICO E 1:25
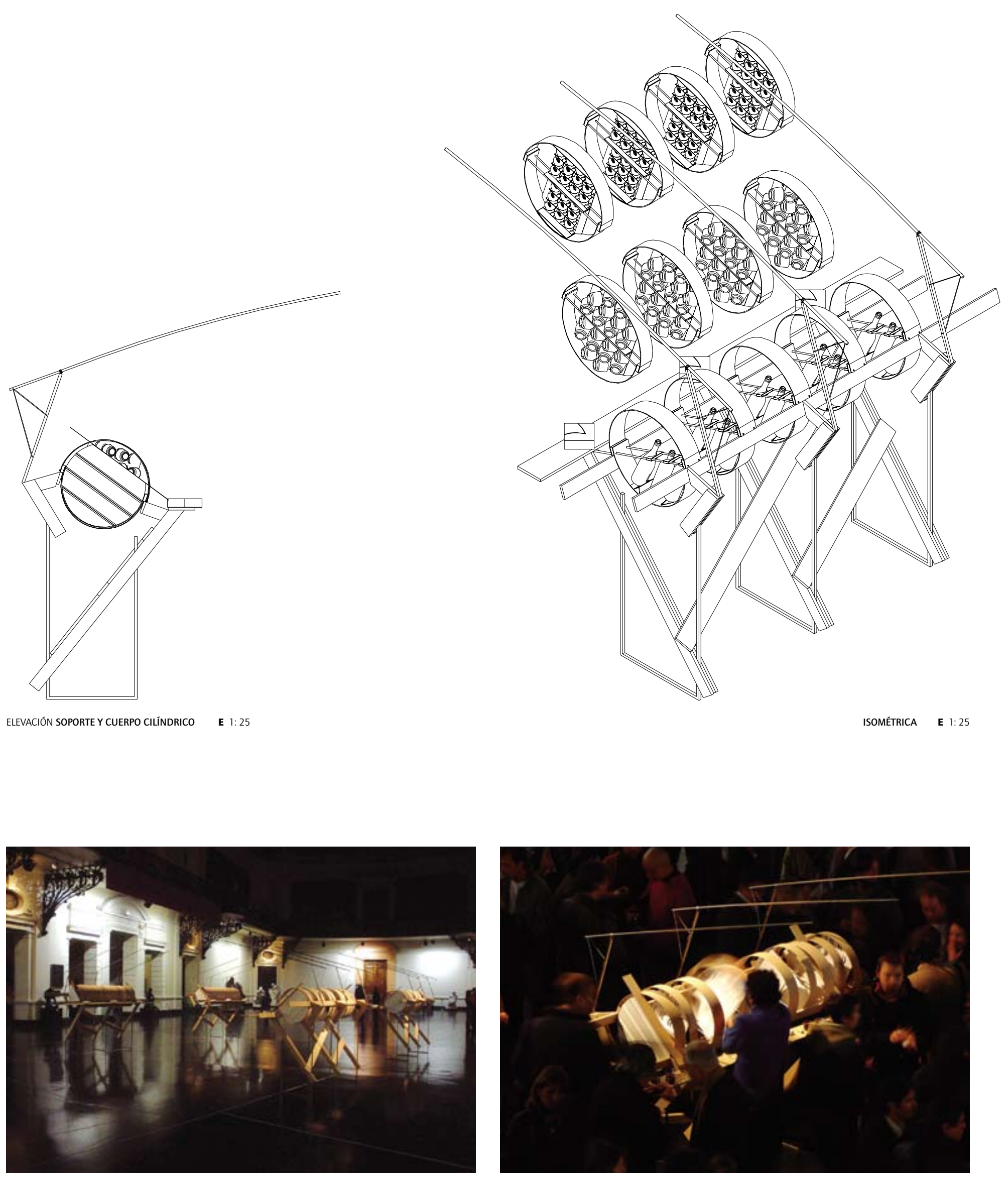